\title{
Relación médico-paciente percibida por usuarios de consultorios externos de un hospital de Lima, Perú
}

\author{
Cinthya Mendoza ${ }^{1}$, Claudia Ramos-Rodríguez ${ }^{2}$, Ericson Gutierrez ${ }^{1,3}$
}

\section{RESUMEN}

Objetivo: Describir la relación médico-paciente (RMP) y determinar los factores asociados a una buena percepción de está por los pacientes que acuden a consultorios externos del Hospital Carlos Lanfranco La Hoz (HCLLH) del Ministerio de Salud (MINSA), período mayo 2014.

Material y Métodos: Estudio transversal-comparativo, realizado en pacientes que acuden a consulta externa de especialidades médicas. Se utilizó el cuestionario estructurado semicerrado, llamado PREMEPA. Los cálculos estadísticos fueron realizados con un nivel de confianza del $95 \%$.

Resultados: Un total de 179 pacientes completaron la encuesta, el 64,8\%(116) fueron mujeres, el promedio de la edad fue de 42,1 $\pm 11,9$ años. El $23 \%$ (41) de los usuarios percibieron una RMP buena, el $52 \%$ (93) regular y el $25 \%$ (45) mala. Las variables finalmente asociadas con una buena RMP fueron tener entre 18-35 años (OR = 8,1 [IC95\%; 1,6-40,1), haber elegido a su médico $(O R=20,5$ [IC95\%; 5,1-82,5) y ser atendido por una médico mujer (OR = 3,1 [IC 95\%; 1,01-9,3]).

Conclusiones: Existe un bajo porcentaje $(23 \%)$ de pacientes que perciben una buena relación médico paciente. El elegir a su médico, ser paciente joven y ser atendido por una médico mujer influyen en tener una buena RMP.

Palabras clave: Relaciones Médico-Paciente, Aceptación de la Atención de Salud, Satisfacción del Paciente. (Fuente: DeCS BIREME).

\section{Physician-patient relationship perceived by outpatients at a hospital in Lima, Peru}

\section{ABSTRACT}

Objective: To describe the Physician-patient relationship and to determine the factors associated with a good perception of it by the patients that attend ambulatory care in the Carlos Lanfranco La Hoz Hospital (CLLHH) of the Health Ministry (MINSA), during May 2014.

Material and Methods: Transversal-comparative study performed with patients that attend ambulatory care of medical specialties. In this study, a structured semi closed questionnaire called PREMEPA was used and statistical calculations were made with $95 \%$ confidence level.

Results: A total of 179 patients completed the questionnaire. Of those, $64.8 \%$ (116) were women, the average age was 42,1 $\pm 11,9$ years old. $23 \%$ (41) of the patients perceived a good physician-patient relationship, $52 \%$ (93) regular and $25 \%(45)$ a bad relationship. The variables finally associated with a good physician -patient relationship were having between 18-35 years old $(\mathrm{OR}=8,1$ [IC95\%; 1,6-40,1), personally choosing their physicians ( $O R=20,5[$ IC $95 \% ; 5,1-82,5)$, and be attended by a female physician (OR = 3,1 [IC 95\%; 1,01-9,3]).

Conclusions: There is a low percentage $(23 \%)$ of patients that perceive a good physician -patient relationship. Choosing their physician, being a young patient and be attended by a female physician influenced in having a good physician-patient relationship.

Keywords: Physician-Patient Relations, Patient Acceptance of Health Care, Patient Satisfaction. (Source MeSH NLM)

\footnotetext{
${ }^{1}$ Facultad de Medicina Humana, Universidad de San Martin de Porres. Lima-Perú.

${ }^{2}$ Servicio de Anatomía Patológica. Hospital General Universitario de Ciudad Real. España.

3 Unidad de Análisis y Generación de Evidencias en Salud Pública, Instituto Nacional de Salud. Lima-Perú.
} 


\section{INTRODUCCIÓN}

La atención en el proceso salud-enfermedad intervienen dos grandes actores sociales, por un lado el paciente y su familia, y por otro los proveedores de salud. Los médicos constituyen uno de los grupos profesionales que brinda atención a la salud de la población y tiene un papel crucial en este ejercicio(1).

La relación médico paciente es aquella interacción que se establece entre el médico y el paciente con el fin de devolverle a éste la salud, aliviar su padecimiento y prevenir la enfermedad (1). Según Perales, la relación médico-paciente es la base del acto médico y una de las mayores fuerzas recuperadoras de la salud (2).

Cuando se establece empatía, la toma de información se facilita, se posibilitan diagnósticos más precisos, se incrementa la adherencia terapéutica, la satisfacción del paciente y se hace menos probable que el paciente desee cambiar de terapeuta, mientras que el uso del tiempo de la consulta se torna más eficiente (3).

La actual mercantilización de los servicios de salud es motivo de preocupación por los efectos negativos que pueden provocar en la relación médico paciente (4). La búsqueda del lucro por parte de empresas privadas puede reducir los tiempos de atención del Médico así como la calidad de atención (5).

En la actualidad nuestro país vive un acelerado aumento de la demanda de atenciones en salud, lo cual afecta a diversos prestadores de salud, sean establecimientos de Seguro Social de Salud (ESSALUD), MINSA/Gobiernos Regionales, Fuerzas Armadas o privados, producida entre otras cosas por la transición demográfica.

Asimismo, debido al aumento de las tecnologías de información, los pacientes están cada vez más empoderados con su salud y las decisiones que se tomen respecto a ella (6), por lo tanto es de vital importancia para las instituciones prestadoras de salud conocer la percepción de los pacientes sobre la relación que se establece con los médicos, con el fin de brindar medidas correctivas cuando se requieran.

En función a lo anteriormente expuesto se plantea el siguiente estudio de investigación que tiene como objetivo describir la relación médico-paciente y determinar los factores asociados a una buena percepción de esta por los pacientes que acuden a consultorios externos del hospital Carlos Lanfranco La Hoz (HCLLH) del Ministerio de Salud (MINSA), período mayo 2014.

\section{MATERIALES Y MÉTODOS}

Estudio transversal comparativo, realizado en pacientes que acuden a consulta en los consultorios externos de especialidades médicas del Hospital Nivel II-2 Carlos Lanfranco La Hoz (HCLLH) del distrito de Puente Piedra, Lima, durante el mes de mayo 2014.

Para el cálculo de muestra en este estudio se utilizó la fórmula para estimar proporciones en poblaciones finitas, considerando una prevalencia de adecuada relación médico paciente de 0,50 , un nivel de confianza de $95 \%$ y un error de $8 \%$. Durante el año 2013, el número de atenciones ambulatorias fue de 120945 por lo que se requirió un tamaño maestral mínimo de 156 personas. Se realizó un muestreo sistemático de arranque aleatorio. Se escogió un número al azar del 1 al 5 , posteriormente se entrevistó los pacientes en intervalos de 5 cada vez que salían de los consultorios.

Se incluyeron a pacientes mayores de edad que voluntariamente aceptaban participar, se excluyeron a pacientes con alguna condición clínica que afecte su lucidez, o que se negaban a participar del estudio.

Se utilizó el cuestionario estructurado semicerrado, llamado PREMEPA (percepción de la relación médicopaciente), diseñado y validado por Ramos C. (7) El cuestionario está conformado por tres bloques, el primero es denominado "Percepción de la relación médico paciente", el segundo "Datos generales del 
médico", y el tercero "Datos generales del paciente".

Para determinar la percepción de la relación médicopaciente, se sumaron las variables de las preguntas que evaluaban las actitudes y características del profesional percibidas en la consulta en cuanto a respeto y cortesía, disponibilidad de escuchar, comprensión, sensibilidad humana así como el grado de confianza en su capacidad profesional y la buena explicación de su enfermedad.

Para construir los rangos de la percepción de la RMP, se utilizó la fórmula de statones, con lo cual se establecieron 3: RMP buena $\geq 14,4$; RPM regular 7,614,$4 ;$ y RMP mala $\leq 7,6$.

Con el fin de buscar factores asociados, se consideró como variable dependiente una adecuada RMP y como variables independientes las características del médico tratante y las variables sociodemográficas de los pacientes.

Se realizó inicialmente estadística univariada y bivariada con la prueba chi cuadrado de Pearson para buscar asociaciones entre las variables independientes y una adecuada RMP. Posteriormente se realizó un análisis multivariado. Para dicho análisis se utilizó el modelo de regresión logística condicional, se estimó el odds ratio (OR) y sus intervalos de confianza. Los cálculos fueron realizados con un nivel de confianza del 95\%. La participación en el estudio fue anónima y voluntaria previo consentimiento verbal, se aseguró el anonimato y la confidencialidad de los pacientes. El estudio fue aprobado por el comité de ética de la Universidad de San Martín de Porres y tuvo autorización formal por parte del hospital donde se realizó el estudio.

\section{RESULTADOS}

Un total de 179 pacientes completaron la encuesta, el $64,8 \%$ (116) fueron mujeres, el promedio de la edad fue de 42,1 $\pm 11,9$ años. En su mayoría los pacientes estuvieron en el rango de edad de $36-50$ años $(41,3 \%)$, tuvieron una educación secundaria completa $(60,9 \%)$ y fueron pacientes continuadores en la consulta $(50,8 \%)$.

Tabla 1. Características sociodemográficas de los pacientes

\begin{tabular}{|cc} 
Edad del Paciente & N $(\%)$ \\
$18-35$ & $56(31,3)$ \\
$36-50$ & $74(41,3)$ \\
$51-65$ & $49(27,4)$ \\
Total & \\
Sexo del Paciente & \\
Femenino & $116(64,8)$ \\
Masculino & $63(35,2)$ \\
Máximo nivel educativo & $17(9,5)$ \\
Primaria & $109(60,9)$ \\
Secundaria & $53(29,6)$ \\
Superior & $49(27,4)$ \\
Tipo de paciente & $91(50,8)$ \\
Paciente nuevo & $39(21,8)$ \\
Continuador &
\end{tabular}

En cuanto a las características del médico y de la atención médica recibida, el $83 \%$ (149) de las atenciones fueron realizadas por médicos varones, el $57 \%$ (103) de los médicos fueron considerados de "mediana edad" por parte de los pacientes. El 11,2\% (20) de los pacientes eligió a su médico para la consulta.

Tabla 2. Características del médico y de la atención médica recibida

$\begin{array}{cc}\text { Sexo del médico } & \text { N }(\%) \\ \text { Femenino } & \\ \text { Masculino } & 30(16,8) \\ \text { Edad del médico } & 149(83,2) \\ \text { Adulto Joven } & \\ \text { Mediana edad } & 60(33,5) \\ \text { Adulto mayor } & 103(57,5) \\ \text { Noted eligió a su médico } & 16(8,9) \\ \text { Sí } & 159(88,8) \\ \text { Número de consultas anteriores } & 20(11,2) \\ \text { Ninguna } & 92(51,4) \\ 1-4 & 79(44,1) \\ \text { Más de } 4 & 8(4,5)\end{array}$


En cuanto a la percepción de la relación médico paciente, se aprecia que el promedio de la percepción de la RMP fue de 10,9 4,5 [Rango 2-21], de un máximo posible de 21 , al categorizar esta percepción se aprecia que el $23 \%$ (41) de los usuarios percibieron una RMP buena, el 52\% (93) regular y el $25 \%$ (45) mala.

Figura 1. Percepción de la relación médico paciente por parte de los usuarios

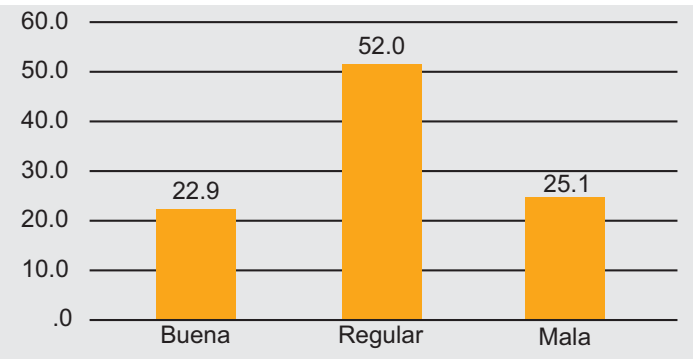

En cuanto a las dimensiones de la percepción de la RMP se aprecia que la dimensión con mayor puntaje fue el respeto y cortesía, seguida por la disponibilidad a escuchar y la explicación de la enfermedad (Tabla 3). El 79,3\% (142) de los pacientes no recomendaría a un familiar o amigo atenderse con el mismo médico con el que realizó la consulta.

Tabla 3. Dimensiones de la relación médico paciente

\begin{tabular}{ccccc} 
Dimensiones & $\begin{array}{c}\text { Totalmente } \\
\text { N }(\%)\end{array}$ & $\begin{array}{c}\text { Medianamente } \\
\text { N (\%) }\end{array}$ & $\begin{array}{c}\text { Muy poco } \\
\text { N (\%) }\end{array}$ & No N $(\%)$ \\
\hline Respeto y Cortesía & $41(22,9)$ & $76(42,5)$ & $44(24,6)$ & $18(10,1)$ \\
Disponibilidad a escuchar & $28(15,6)$ & $64(35,8)$ & $68(38,0)$ & $19(10,6)$ \\
Comprensión & $15(8,4)$ & $74(41,3)$ & $60(33,5)$ & $30(16,8)$ \\
Sensibilidad & $10(5,6)$ & $83(46,4)$ & $63(35,2)$ & $23(12,8)$ \\
Confianza & $14(7,8)$ & $74(41,3)$ & $78(43,6)$ & $13(7,3)$ \\
Explica enfermedad & $26(14,5)$ & $75(41,9)$ & $62(34,6)$ & $16(8,9)$ \\
Explica el procedimiento & $23(12,8)$ & $88(49,2)$ & $52(29,1)$ & $16(8,9)$ \\
\hline
\end{tabular}

En el análisis bivariado, la edad del paciente, el ser paciente mujer, haber elegido a su médico, ser atendido por un médico joven y por una médico mujer estuvieron asociados a una buena relación médico paciente.

En el modelo de regresión logística, se aprecia que las variables finalmente asociadas con una buena RMP fueron tener entre 18-35 años (OR = 8,1 [IC95\%; 1,6$40,1)$, haber elegido a su médico (OR = 20,5 [IC95\%; $5,1-82,5)$ y ser atendido por una médico mujer $(O R=$ 3,1 [IC 95\%; 1,01-9,3]).
Tabla 4. Factores asociados a una buena relación médico paciente

\begin{tabular}{|c|c|c|c|c|c|}
\hline \multirow[b]{2}{*}{ Variables } & \multirow[b]{2}{*}{$N(\%)$} & \multicolumn{2}{|c|}{ Análisis bivariado } & \multicolumn{2}{|c|}{ Análisis multivariado } \\
\hline & & OR [IC 95\%] & Valor $\mathrm{p}$ & OR [IC 95\%] & Valor $\mathrm{p}$ \\
\hline \multicolumn{6}{|c|}{ Edad del paciente } \\
\hline $18-35$ & $25(44,6 \%)$ & - & $<0,0001$ & $8,1[1,6-40,1]$ & 0,01 \\
\hline $36-50$ & $14(18,9 \%)$ & - & & $3,1[0,6-15,4]$ & 0,167 \\
\hline $51-65$ & $2(4,1 \%)$ & - & & 1 & \\
\hline \multicolumn{6}{|c|}{ Paciente mujer } \\
\hline Sí & $34(29,3 \%)$ & $3,3[1,4-8]$ & 0,006 & $1,3[0,4-3,7]$ & 0,67 \\
\hline No & $7(11,1 \%)$ & & & 1 & \\
\hline \multicolumn{6}{|c|}{ Educación superior } \\
\hline Sí & $14(26,4 \%)$ & $1,3[0,6-2,8]$ & 0,469 & & \\
\hline No & $27(21,4 \%)$ & & & & \\
\hline \multicolumn{6}{|c|}{ Eligió a su médico } \\
\hline Sí & $17(85 \%)$ & $31,9[8,7-117,2]$ & $<0,0001$ & $20,5[5,1-82,5]$ & $<0,0001$ \\
\hline No & $24(15 \%)$ & & & 1 & \\
\hline \multicolumn{6}{|c|}{ Se atendió anteriormente } \\
\hline Sí & $21(24,1 \%)$ & $1,2[0,57-2,3]$ & 0,703 & & \\
\hline No & $20(21,7 \%)$ & & & & \\
\hline \multicolumn{6}{|c|}{ Médico joven } \\
\hline Sí & $19(31,7 \%)$ & $2[1,0-4,2]$ & 0,048 & $2,4[0,9-6,2]$ & 0,067 \\
\hline No & $22(18,5 \%)$ & & & 1 & \\
\hline \multicolumn{6}{|c|}{ Médico mujer } \\
\hline Sí & $13(43,3 \%)$ & $3,3[1,4-7,6]$ & 0,004 & $3,1[1,01-9,3]$ & 0,045 \\
\hline No & $28(18,8 \%)$ & & & 1 & \\
\hline
\end{tabular}

\section{DISCUSIÓN}

En el presente estudio apreciamos que un bajo porcentaje (23\%) de pacientes percibe una buena relación médico-paciente en el hospital II-2 Carlos Lanfranco La Hoz (HCLLH). En nuestro país, encontramos escasos estudios que abordan la percepción de la relación médico-paciente por parte de los usuarios, con los cuales comparar los resultados obtenidos. Un estudio previo realizado en el Hospital Carrión de Callao, el cual utilizó el mismo instrumento, demostró que el 92,3\% de los pacientes que acudían a los consultorios externos de un departamento de medicina, percibieron una adecuada relación médico-paciente (7).

De manera similar en un estudio realizado en 6 centros de atención primaria de Madrid-España, se encontró una alta tasa de satisfacción con la relación médico- paciente por parte de los usuarios (8). 
Los ítems de más bajo puntaje fueron en las dimensiones de confianza, sensibilidad y comprensión. Estudios previos demuestran que en el Perú existen deficiencias en la formación de médicos en habilidades clínicas como la evaluación de la compresión del paciente, el manejo de las emociones y la comunicación no verbal (9).

La comunicación con el paciente es una competencia esencial que tiene que tener el médico para su trabajo diario, así mismo los pacientes perciben que un médico que los escucha puede recopilar adecuadamente datos clínicos para un adecuado diagnóstico, actúa como un agente terapéutico y fortalece la relación médico-paciente $(10,11)$. Lamentablemente el escaso tiempo con el que cuentan los médicos para cada atención disminuye la posibilidad de una adecuada comunicación con su paciente, situación que es expresada por los propios médicos en estudios previos (9).

En el análisis de los factores asociados apreciamos que los pacientes que eligieron a su médico tuvieron muchas más probabilidad de tener una buena relación médico-paciente. En este caso consideramos que el paciente ya entabló previamente una relación de cordialidad con su médico tratante, por lo cual emite puntajes altos en cada uno de los ítems evaluados. Por el contrario los pacientes que acuden por primera vez, no perciben una buena relación con los médicos tratantes.

Los pacientes más jóvenes también tienen una mejor percepción de la relación médico paciente, la asociación entre el grupo etáreo y la percepción de la relación médico paciente ya fue descrito en un estudio similar (7). Es posible que los pacientes jóvenes acudan a la consulta por dolencias de menor complejidad o por la búsqueda de algún certificado de salud. Por el contrario los pacientes de mayor edad acuden por dolencias de mayor complejidad, por lo que necesiten mayor tiempo de atención y tienen mayores expectativas con la atención del médico. Por lo tanto una atención rápida y no tan exhaustiva sería suficientes para tener una adecuada relación médico paciente en el caso de un paciente joven que acude por una dolencia menor.
Ser atendido por un médico mujer también fue un factor asociado a una adecuada relación médicopaciente. Un estudio previo demostró que las internas de medicina tenían un mayor nivel de empatía que sus pares varones (12). Podemos asumir que los pacientes perciben que las mujeres tienen una mayor sensibilidad y empatía con los pacientes, esta condición del personal de salud femenino ha sido reportada en literatura(13).

Se puede considerar una limitación del estudio el poco número de muestra. En este sentido, el máximo margen de estimación de error aceptable para el muestreo de un estudio suele estar entre un 5\% y $10 \%$ (14), por lo que en este caso se utilizó un valor de $8 \%$. Debido a limitaciones de tiempo y de recursos para obtener un mayor número de encuestas no tomamos el valor que nos daría el mayor número de muestra, que este caso sería de $5 \%$. Sin embargo el número de pacientes que acuden a los consultorios de este hospital es relativamente pequeño, por lo que la muestra calculada puede proveer de estimaciones adecuadas.

Otra limitación es que el estudio fue realizado en un solo establecimiento de salud, por lo que estos resultados no se podrían generalizar a otros establecimientos del MINSA, sin embargo puede servir de referencia para próximos estudios que se realicen en esta misma temática.

En conclusión La percepción de la relación médico paciente fue principalmente regular. Ser un paciente joven, elegir a su médico y ser atendido por una médico mujer estuvieron asociados a una buena percepción de la relación médico paciente.

Estos resultados podrían servir para plantear medidas de intervención para mejorar la percepción de la relación médico-paciente en este hospital. Así mismo podrían ser tomados como línea de base para medir el impacto de las fututas intervenciones a realizar. 


\section{REFERENCIAS BIBLIOGRÁFICAS}

1. Mastache P. Percepción de la Comunicación en la relación Médico-Paciente por los Médicos pasantes en Servicio Social de la Universidad Autónoma de Querétaro. [Tesis para optar el grado de Magister en Ciencias de la Salud]. Querétaro-México: Universidad Autónoma de Querétaro; 2011.

2. Sánchez GJM, Rivera CAE, Hernández GLE, et al. La Relación médico paciente y la comunicación deben estar asociados a la Ética Médica. Revista Calimed 2002; 8(1):21-27.

3. Perales, Alberto. "El acto médico: Criterios de definición y límites”. Diagnóstico 2001; 40:46-50.

4. Calvo A. La relación Médico-Paciente. Rev Med Hered 2006; 17 (4): 187.

5. Dorr Goold S, Lipkin M Jr.The doctor-patient relationship: challenges, opportunities, and strategies. J Gen Intern Med 1999;14 Suppl 1:S26-33.

6. Caballero C. E-pacientes y la relación médico-paciente. Salud Uninorte 2013; 29 (2): 6-7.

7. Ramos C. Percepción de las relaciones médico-paciente, por parte de los usuarios externos de un departamento de medicina. An Fac med 2008; 69(1):12-6

8. Martín-Fernández J, del Cura-González MI, Gómez-Gascón T, Fernández-López E, Pajares-Carabajal G, Moreno-Jiménez B. [Patient satisfaction with the patient-doctor relationship measured using the questionnaire (PDRQ-9)]. Aten Primaria 2010; 42(4):196-203.

9. Sogi C, Zavala S, Oliveros M, Salcedo C. Autoevaluación de formación en habilidades de entrevista, relación médico paciente y comunicación en médicos graduados. An Fac Med Lima 2006; 67(1): 30-7

10. Ramírez J, Ocampo R, Pérez I, Velázquez D Yarza E. La importancia de la comunicación efectiva como factor de calidad y seguridad en la atención médica. ACTA MÉDICA GRUPO ÁNGELES 2011; 9: 167-74.

11. Moore P, Gómez G, Kurtz S. [Doctor-patient communication: one of the basic competencies, but different]. Aten Primaria 2012;44(6):358-65.

12. Remón-Torres $M$, Remón-Torres $M$, Tataje-Lavanda $M$. Características del nivel de empatía hacia el paciente en internos de medicina de dos hospitales nacionales. Rev méd panacea. 2013; 3(1): 2-5.

13. Quiñones A. La aportación de la enfermera en la relación médico-paciente: un plus de intuición, cercanía y competencia. Revista de Humanidades UP 2008; 1(1): 11-8.

14. Superintendencia de Salud de Chile. Indicadores de calidad: Manual de muestreo para prestadores. Disponible en: http: / / www.supersalud.gob.cl/observatorio/575/articles6358_recurso_1.pdf
Fuentes de Financiamiento

Este artículo ha sido financiado por los autores.

\section{Conflicto de interés}

Los autores declaran no tener ningún conflicto de interés.

\section{Correspondencia:}

Ericson Leonardo Gutierrez Ingunza.

Dirección: Calle Cápac Yupanqui 1400. Jesús María. Lima 11, Perú Teléfono: (511) 965032850

Correo electrónico: eringunza@yahoo.es

Recibido: 03 de diciembre de 2015 Aprobado: 11 de febrero de 2016 